\title{
Comparison Analysis of proposed DPPH Protocol for Wireless Body Area Network
}

\author{
Madhumita Kathuria \\ Department of \\ Computer Engineering \\ YMCA University of \\ Science and Technology \\ Faridabad, Haryana, India
}

\author{
Sapna Gambhir \\ Department of \\ Computer Engineering \\ YMCA University of \\ Science and Technology \\ Faridabad, Haryana, India
}

\begin{abstract}
Given the emergence of pervasive computing, Wireless Body Area Networks (WBANs) will play an increasingly important role in future critical system like Healthcare. Wireless Body Area Networks are consists of many inexpensive sensor nodes with limited energy and computational resources and memory. In this paper, a survey of existing projects is done to identify all the issues related to the healthcare system. Some of the major challenges in these networks are heterogeneous traffic handling, loss and delay free data transmission in the frequently changing environment. Proper traffic handling with dynamic prioritization concept makes the healthcare system more effective in frequently changing and time critical healthcare environment. A number of existing protocols for this have been proposed in the past few years. This paper compares them with the proposed Dynamic Priority based Packet Handling (DPPH) protocol. The evaluation is done using the NS-2.35 simulator, which provides a convenient platform. The performance metrics under investigation are the packet delivery ratio, packet loss ratio, end-to-end delay, and throughput. We found that the DPPH protocol can utilize the resources very well while keeping the throughput high and helping in controlling the packet loss and delay.
\end{abstract}

\section{Keywords}

Dynamic, Priority, Throughput, Delay, Packet Delivery, Wireless Body Area Network.

\section{INTRODUCTION}

Healthcare is becoming a vital part of living being and reveals requirements of an effective response to medical emergencies. In this paper, we provide thorough discussions on the existing issues in healthcare systems and feasible techniques for these issues. Additionally, we exhibit the conflicting goals through an exemplary comparison scenario, and a sound solution is proposed to leverage these conflicts. The main target of current healthcare systems is to solve critical condition detection, emergency response, remote and unobstructed monitoring of patients. The requirement of these problems can be resolved by integration of expert medical knowledge with ubiquitous Wireless Body Area Network (WBAN). Therefore, this area is becoming a new era for the researcher in the foresee future.

So far, several projects have been introduced in this regard, for example, CodeBlue, MobiHealth, UbiMon, Alarm-Net, MEDiSN etc. Basically, the main objectives of these projects are to provide reliable communication, data rate, routing, and Quality of Service (QoS). The CodeBlue [1] was designed to serve people in emergency cases, patient rehabilitation and disaster, along with real-time monitoring and location tracking for an extended period of time. It takes care of in-network filtering, aggregation and handoff services. It provides a query management policy, which allows the medical expert to request data in emergency cases, and also provides a filtering facility. The core foundation of CodeBlue is its Adaptive Demand Driven Multicast Routing (ADMR) protocol, which establishes multicast paths. A forwarder simply rebroadcasts any coming messages, using duplicate suppression in order to avoid multiple transmissions. MobiHealth [2] performs incessant health monitoring with the help of GPRS and UMTS cellular networks. It timely senses the patient's body medical statics and broadcast it to the client. The client segregates the data and sends to the processing server. The aim of UbiMon [3] is to provide unobtrusive and distributed mobile monitoring capture each transient event. UbiMon illustrates a logical cross-disciplinary integration of different expertise of the fields, bringing together electronics, computing, medicine. ALARM-Net [4] is designed as a heterogeneous network for monitoring environmental and physiological data. It incorporates a Circadian Activity Rhythm (CAR) (which is full day routine of activity of an individual) analysis module that learns the patterns of daily life and influences the system for power management and privacy. Additionally, CAR decides which sensors should be kept active and which can be temporarily disabled. Its querying security feature allows authorized person to access the information. The Privacy Manager comprised of a gateway called AlarmGate. The AlarmGate has three major functional components: the Context Manager, the Request Authorizer, and the Auditor. The Context Manager collects and maintains the context objects about users and the environment. All queries received at the Query Manager are forwarded to the Request Authorizer. It takes decisions regarding access by consulting the system's privacy policies and context objects of the query subject. The Auditor maintains a trace of the access request in an audit trail, including the authorization decision made for each request (i.e. granted or denied). The Security Manager access is limited to legitimate users. MEDiSN in [5] was designed for patient monitoring and disasters. MEDiSN uses a particular wireless backbone, treats all packets equally and deals with priorities at the application layer. It piggybacks alerts to all of their outgoing messages. Doing so, it increases the probability of delivering these alerts despite faults such as lost packets and link failures. It is having multiple physiological monitors (PMs), which are mobile, temporarily storing sensed data and transmitting it (after encrypting and signing the sensed data) to the relay points (RPs). MEDiSN incorporates different stationary RPs that are self-organized into a bidirectional routing tree and forwards PM data to the gateways and vice versa. The RPs uses a collection tree routing protocol to forward their measurements to the gateway. It is connected 
with a database that stores and presents data to authenticated GUI clients.

In this paper, we describe the working details of Dynamic Priority Based Packet Handling (DPPH) protocol designed for the architecture given in [15]. This is a novel protocol, which classifies heterogeneous traffics and assigns priority dynamically. It also takes care of emergency conditions and issue alerts against them. We also compare the performance of DPPH with existing protocols in terms of packet delivery ratio, packet loss ratio, delay, and throughput.

This paper is organized as follow. Section 2 illustrates the related work. Section 3 gives the working details of proposed protocol. Section 4 represents the comparison analysis. Section 5 implements the performance metrics through simulation. Section 6 provides the conclusion part.

\section{RELATED WORK}

This section provides a detailed review of the various existing protocols used in the WBAN domain by discussing their strengths and weaknesses.

\subsection{PCCP}

Priority-based Congestion Control Protocol (PCCP) introduced in [7], is an upstream congestion control protocol for WSNs. It measures the degree of congestion as the ratio of packet inter-arrival time to the packet service time. Based on the level of congestion degree and node priority index, PCCP utilizes a cross-layer optimization and imposes a hop-by-hop mechanism to control congestion. PCCP achieves efficient congestion control and flexible weighted fairness for both multipath routing and single-path routing.

PCCP uses congestion degree, which is defined as the ratio of service time over inter-arrival time, to more accurately measure congestion at each sensor node. PCCP consists of three components: Implicit Congestion Notification (ICN), Intelligent Congestion Detection (ICD), and Priority-based Rate Adjustment (PRA). ICD detects congestion based on packet inter-arrival time and packet service time. The joint participation of inter-arrival and service times in ICD reflects the current congestion level and which further provide helpful and rich congestion information. PCCP uses implicit congestion notification to avoid transmission of additional control messages and that leads to improving energyefficiency. In ICN, congestion information is piggybacked in the header of data packets and further broadcast to child nodes, when packets are forwarded by their parent nodes towards the sink. Finally, PCCP designs a novel priority-base rate adjustment algorithm (PRA) employed in each sensor node in order to guarantee both flexible weighted fairness and throughput, where each sensor node is given a priority index. PRA is designed to guarantee that: (1) The node with higher priority index gets more bandwidth; (2) The nodes with the same priority index get equal bandwidth. (3) A node with sufficient traffic gets more bandwidth than one that generates less traffic.

PCCP achieves high link utilization and flexible fairness with small buffer occupancy; therefore, it improves energyefficiency and reduces delay and loss.

This protocol does not entertain congestion avoidance procedure. It performs poorly in providing relative priority in case of occurring different congestion situations. In the case of low congestion, PCCP increases the scheduling and data rate of all traffic sources without paying attention to their priority index. In the case of high congestion, it decreases the sending rate of all traffic sources. Another problem of PCCP is that it considers the only geographical priority and does not support multi-sensor with dynamic priority (i.e. overall health status) which are necessary for healthcare systems.

\subsection{LACAS}

Learning Automata-Based Congestion Avoidance Algorithm in Sensor Networks (LACAS) [9] mitigates the congestion problem by placing some simple autonomous learning machines, called "automata" at each of the nodes of the network. The nodes are capable of controlling the rate of flow of data at the intermediate nodes.

The automaton policy present at each node "learns" from the past behavior and chooses a better data flow rate that tries to avoid congestion. Instead of updating the data flow rate at the source node, the intermediate nodes themselves can be fed with the knowledge of the traffic flow characteristics so that congestion can be avoided even before it occurs. The LACAS tries to make both the packet arrival rate and the packet service rate equal, preventing any kind of queuing at the nodes to a large extent and, hence, reduce delay.

This approach helps to increase the efficiency of the network by optimizing the unnecessary consumption of valuable network resources such as the residual energy at the nodes and the link capacities. With LACAS, successful packet delivery is quite high because an automaton at a node is selecting the rate that is optimum with respect to the number of packets dropped. This leads to reduced queuing of packets, reduced levels of collision and, in effect, increased throughput.

This protocol is not able to provide fair bandwidth allocation, scheduling, and treats each node equally. It is unable to differentiate heterogeneous node or traffic, due to lack of prioritization policy.

\subsection{OCMP}

The Optimized Congestion Management Protocol for Healthcare Wireless Sensor Networks (OCMP) [12] solves the problem of congestion by a proper adjustment of the sending rate of each child node using an optimization function. The proposed optimization function adjusts the sending rate of each child node based on the available bandwidth and the dynamic priority level of each child node.

It consists of two stages. The first stage tries to avoid congestion by using an Active Queue Management (AQM) policy. It decides either to accept or to drop an incoming packet, according to a packet drop probability. If the incoming packet is accepted, then in the second stage, three mechanisms are used to control possible congestion, namely, Automata based Congestion Detection (ACD), Implicit Congestion Notification (ICN), and Optimized Rate Adjustment (ORA). The ACD detects congestion by a three-state machine and virtual queue status. The output of $\mathrm{ACD}$ is the congestion level $(0 \leq C L \leq 1)$. If $C L$ is more than a specific threshold, the optimized rate adjustment (ORA) procedure will be called. This procedure will resolve an optimization problem and will obtain new share rates for child nodes as well as local source traffic. It uses child priority (CHP) and Available Bandwidth $(\mathrm{AB})$ as the input parameters for the optimization function (i.e. $\mathrm{AB}=1-\mathrm{CL}$ ). This protocol uses the implicit Congestion Notification (ICN) which piggybacks congestion information in the header part of data packets and avoids sending additional control messages which consequently, causes an improvement in energy-efficiency. 
The Active Queue Management (AQM) scheme tries to control congestion and to improve network performance such as network delay, link utilization, packet loss rate, and system fairness.

The proposed algorithm uses a flexible procedure for queue management. This procedure uses a single physical queue which is divided into several virtual queues to store the input packets from each child node. It means that a physical queue is shared virtually between $\mathrm{N}$ child node's traffic and one local source's traffic based on their importance and priority. If a child node has more priority, it can use more space for its queue. However, the boundaries between these virtual queues are not constant; when one of the child nodes has a free space in its own virtual queue, others can use the free space if necessary. It assigns dynamic weights to each child node and follows a fair queue management policy. If any child node's queue is likely to be full, then it can use the free space of other child node's queue. OCMP helps to minimize the packet drop and loss rate for high priority traffic classes, reduces starvation for low priority traffic, provides fair scheduling by applying weighted fair queue, utilizes bandwidth in a better way and achieves more throughputs. Optimized rate adjustment mechanism increases the transmission rate when the network becomes underutilized and decreases the transmission rate when the network becomes over utilized.

It tries to solve service classification and prioritization problem to some extent but fails to achieve better performance and utilization of resources.

\section{DPPH PROTOCOL}

Nowadays healthcare applications are becoming a dedicated research area in the vicinity of WBAN, with the requirement of various issues i.e. heterogeneous traffic handling, health data transmission with significant loss and delay etc.

These requirements stimulate us to develop a novel protocol, which can handle heterogeneous traffic in an appropriate way in dynamic WBAN applications. The proposed protocol present in [15], is consists of three units: i) Wireless Body Area Network Unit (WBANU), ii) Controller Unit (CU), and iii) Medical Server Unit (MSU).

\subsection{Wireless Body Area Network Unit}

This unit consists of sensor nodes that sense, and process vital signals, and sends them to CU.

\subsubsection{Data sensing and pre-processing Unit}

After connection establishment, all sensor nodes sense and process vital signals in data sensing and pre-processing unit. This unit manages data and control packets, allocates resources in terms of buffer and bandwidth, and organizes transmission time and data sending rate to each sensor nodes. These values can be changed and updated with time and critical condition of the patient. Proposed architecture provides an efficient and fair utilization of the bandwidth for heterogeneous traffic requirements, as bandwidth allocation in a dynamic environment is a key issue in healthcare WBAN.

\subsubsection{Packet Dispatching Unit}

The main focus of this is to classify, queue and schedule the packets according to the traffic type and sensor priority. The classification unit classifies the sensed packets into real-time or non-real-time data. The queuing unit keeps the real-time bandwidth deficient packets into first in first out (FIFO) based high priority (HP_FIFO) queue and non-real-time packets into FIFO based low priority (LP_FIFO) queue. The scheduling unit schedules the packets according to the proposed 30:10 ratio based policy, where the scheduler serves $30 \%$ packets from HP_FIFO queue and then $10 \%$ packet from LP_FIFO queue. The main motive of this unit is to reduce the delay and starvation problem.

\subsection{Controller Unit}

The main job of this unit is to aggregate, process and handles heterogeneous vital signals and update database accordingly.

\subsubsection{Alerting Unit}

It monitors the variations in sensed data for a particular time interval and calculates the mean deviation of these variations, and checks it against the critical threshold value, if a violation occurs, and then it activates the alert field of the recently received packet and sends it to the classification unit.

\subsubsection{Packet Classification Unit}

The proposed protocol employs a dynamic priority assignment policy to represent packet importance. This unit classifies packets into one of four groups, assigns them priority and serves them accordingly. The Alert packet assigns with priority 1 ; Real-time packet with priority 2 ; On_Demand packet with priority 3 ; and Normal packet with priority 4 .

\subsubsection{Packet Queuing Unit}

It plays an integral role in organizing pending packets. Proposed architecture uses two kinds of Double Ended Priority Queues (DEPQ), one to store high priority packets and another to store low priority packets. This policy overcomes the problem of switching problem and enhances resource utilization in terms of the buffer.

\subsubsection{Packet Scheduling Unit}

The DPPH protocol provides an improved Packet Scheduling approach named as Ratio based Earliest Deadline First (REDF) scheduling. This algorithm tries to reduce the low priority packet starvation as well as the queue starvation problems. REDF reduces the drop rate and starvation rate and enhances the servicing rate by dropping low priority and deadline expired packets.

\subsubsection{Prioritization Unit}

The DPPH protocol delivers a novel prioritization policy to handle critical vital signals. Here all sensor nodes are assigned dynamic priority according to the criticality level of their sensed value. A medical person sets the prioritization field in the header field of control packet as an indication of changes in preset parameters, and assigns new values like dynamic priority of each sensor nodes; range of vital signals; critical threshold; monitoring time etc. All the sensor nodes calculate new data sending rate, transmission time gap, and bandwidth according to sensor nodes new priority. The medical person can also ask for some data in urgent basis, by activating the On_Demand request field in the control packet.

\subsection{Medical Server Unit}

It monitors the received data along with the previous data, takes decisions and updates its database accordingly. If the received data is out of range, then it notifies the medical person by sending the alert message.

To deal with heterogeneity, emergency problem and dynamic changes in the healthcare system, DPPH is developed with a dynamic priority assignment and alerting strategy. Its motto is to fairly transmit packets according to their significance and needs and dynamically utilize all the available resources in terms of buffer and bandwidth. 


\section{COMPARION ANALYIS}

The existing protocols are not able to provide up to mark performance in a frequently changing and heterogeneous healthcare WBANs. The goal of proposed DPPH is to adapt the frequent changes in the WBAN and design a most favorable framework. To cope with heterogeneous traffic and dynamic environment, DPPH dynamically handles traffic and assigns priority. Its main aim is to service packets according to their criticality and dynamically utilize all the available resources. The proposed protocol handles emergency condition depending on criticality level of the vital signal, and by issuing alerts. The DPPH improves the performance of the WBAN by assigning dynamic priority to both node and traffic. An exploration of related issue and solution along with significant comparison is given in Table 1.

Table 1. Comparison table for various protocols.

\begin{tabular}{|c|c|c|c|c|}
\hline Protocol -> & РCCP & LACAS & OCMP & DPPH \\
\hline Resolve Issues & Congestion & $\begin{array}{l}\text { Congestion, and } \\
\text { Energy } \\
\text { Consumption }\end{array}$ & Congestion & $\begin{array}{c}\text { Heterogeneous Packet handling, } \\
\text { and Alerting }\end{array}$ \\
\hline System Layer & Cross-layer, MAC & MAC & MAC & Transport \\
\hline $\begin{array}{l}\text { Data sending } \\
\text { rate (DSR) }\end{array}$ & $\begin{array}{l}\text { Slow start and } \\
\text { priority based }\end{array}$ & Slow start based & $\begin{array}{l}\text { Slow start and } \\
\text { priority based }\end{array}$ & $\begin{array}{l}\text { Quick start and node's priority } \\
\text { based }\end{array}$ \\
\hline $\begin{array}{c}\text { Packet } \\
\text { transmission time } \\
\text { gap (TTG) }\end{array}$ & Fixed & Fixed & Fixed & $\begin{array}{l}\text { Varies according to node's } \\
\text { priority }\end{array}$ \\
\hline $\begin{array}{c}\text { Bandwidth } \\
\text { Allocation }\left(B W_{\text {av }}\right)\end{array}$ & $\begin{array}{l}\text { Depends on sensor } \\
\text { node priority }\end{array}$ & - & $\begin{array}{l}\text { Depends on sensor } \\
\text { node priority }\end{array}$ & Depends on sensor node priority \\
\hline Prioritization & $\begin{array}{l}\text { Node level } \\
\text { prioritization }\end{array}$ & $\begin{array}{l}\text { Treat all node } \\
\text { equally }\end{array}$ & $\begin{array}{c}\text { Static and } \\
\text { Dynamic } \\
\text { prioritization; } \\
\text { Patient and Node } \\
\text { level prioritization }\end{array}$ & $\begin{array}{c}\text { Static and Dynamic based } \\
\text { prioritization; } \\
\text { Both Node and Packet level } \\
\text { prioritization }\end{array}$ \\
\hline Classification & $\begin{array}{l}\text { Service based } \\
\text { classification }\end{array}$ & - & $\begin{array}{l}\text { Service based } \\
\text { classification }\end{array}$ & $\begin{array}{l}\text { Classification based on Packet } \\
\text { type, traffic flow type, } \\
\text { bandwidth, and criticality }\end{array}$ \\
\hline Scheduling & $\begin{array}{l}\text { Weighted Round } \\
\text { Robin queue (WRR) } \\
\text { or WFQ based } \\
\text { scheduling } \\
\end{array}$ & $\begin{array}{l}\text { First Come First } \\
\text { Serve (FCFS) } \\
\text { based scheduling }\end{array}$ & $\begin{array}{l}\text { Weighted fair } \\
\text { queue (WFQ) } \\
\text { based } \\
\text { scheduling }\end{array}$ & $\begin{array}{l}\text { Ratio based Earliest Deadline } \\
\text { First (REDF) scheduling }\end{array}$ \\
\hline Starvation & $\begin{array}{l}\text { Solve queue level } \\
\text { starvation problem }\end{array}$ & - & $\begin{array}{l}\text { Solve queue level } \\
\text { starvation problem }\end{array}$ & $\begin{array}{l}\text { Solve both queue and packet } \\
\text { level starvation problem }\end{array}$ \\
\hline $\begin{array}{l}\text { Performance } \\
\text { metrics }\end{array}$ & $\begin{array}{l}\text { Throughput, Packet } \\
\text { Delivery ratio, Delay, } \\
\text { Fair bandwidth } \\
\text { utilization }\end{array}$ & $\begin{array}{c}\text { Energy } \\
\text { consumption, } \\
\text { Collision, } \\
\text { Throughput, } \\
\text { Packet Delivery } \\
\text { ratio, Drop rate }\end{array}$ & $\begin{array}{l}\text { Throughput, } \\
\text { Packet Delivery } \\
\text { ratio, Delay, Fair } \\
\text { queue and } \\
\text { bandwidth } \\
\text { utilization }\end{array}$ & $\begin{array}{l}\text { Throughput, Packet Delivery } \\
\text { Ratio, Delay, Loss Ratio, Drop } \\
\text { rate, Fair queue and bandwidth } \\
\text { utilization. }\end{array}$ \\
\hline
\end{tabular}

\section{PERFORMANCE EVALUATION}

The proposed protocol is compared with related existing protocols to evaluate the performance of the network in terms of performance metrics i.e. Packet Delivery Ratio (PDR), Packet Loss Ratio (PLR), Delay, and Throughput with respect to variation in a number of nodes. These performance metrics are implemented using the NS-2.35 simulator and graphs are generated using Matlab.

\subsection{Packet Delivery Ratio (PDR)}

The PDR is defined as the ratio of number of received packets at the $\mathrm{CU}$ to the number of generated packets from all sensors. The Fig. 1 shows the comparison of the packet delivery ratio for proposed and existing protocols. 


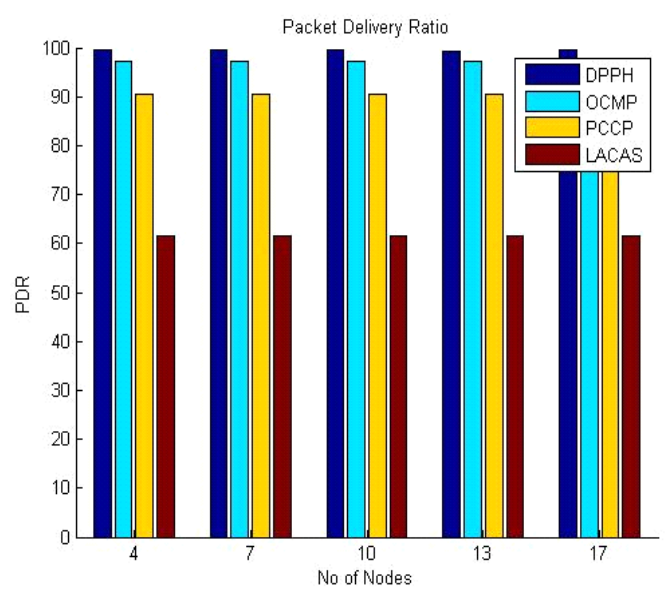

Fig 1: Comparison analysis of PDR for DPPH, OCMP, PCCP, LACAS.

The increase in the PDR in the proposed DPPH protocol shows that higher numbers of packets are delivered as compared to the existing protocols. The PDR of DPPH remains constant for increasing number of nodes.

\subsection{Packet Loss Ratio (PLR)}

The PLR calculates the number of packets lost in the transmission. It is denoted as the ratio of total number of packet loss with respect to total number of packets transmitted. The Fig. 2 shows the comparison of the PLR for proposed and existing protocols.

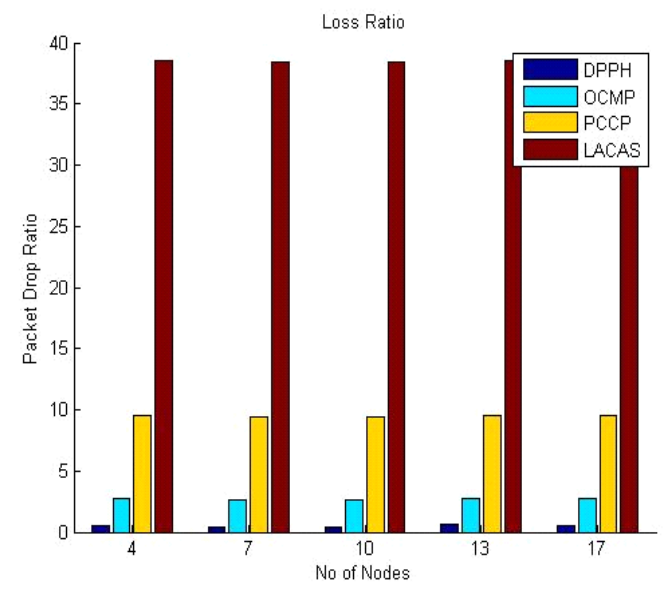

Fig 2: Comparison analysis of PLR for DPPH, OCMP, PCCP, LACAS.

The reduction in packet loss ratio tells the contribution of proposed protocol in dynamic WBAN systems.

\subsection{Delay}

The delay gives the time taken by the packet to transmit from source to destination or the time taken to deliver a packet successfully at the destination. This metric is calculated by subtracting the time at which the packet is transmitted from the sensor node from the time at which the packet arrives to CU. The Fig. 3 shows the comparison of the delay for proposed and existing protocols.

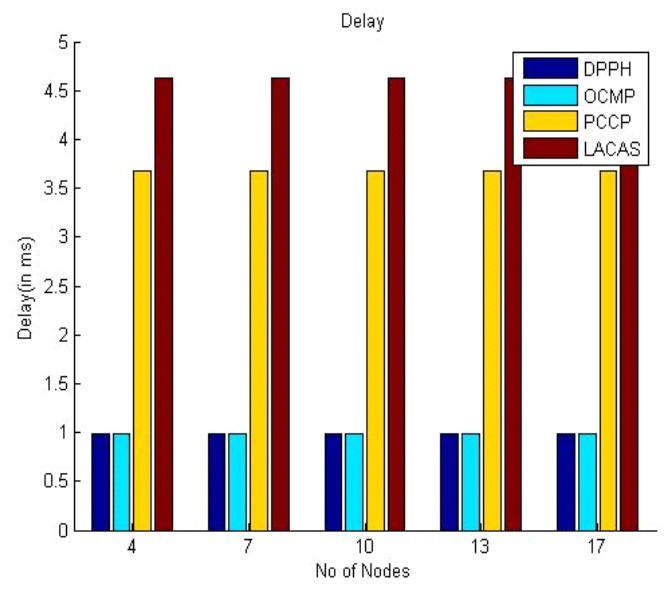

Fig 3: Comparison analysis of Delay for DPPH, OCMP, PCCP, LACAS.

The graph illustrates that there is no impact on delay on increasing the node counts in either of the protocols; it also shows that PCCP \& LACAS both protocol are having higher delay value in comparison to DPPH \& OCMP.

\subsection{Throughput}

It defines the ratio of total number of received packets at destination with respect to the total monitoring time. The Fig.4 shows the comparison of the throughput for proposed and the existing protocol.

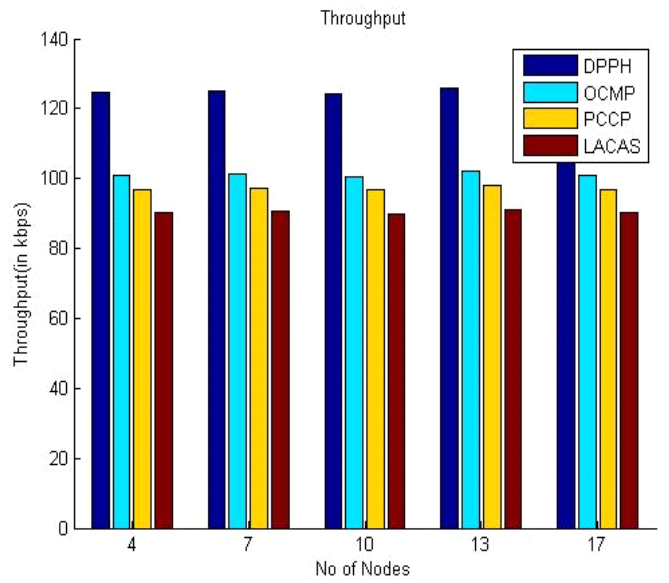

Fig 4: Comparison analysis of Throughput for DPPH, OCMP, PCCP, LACAS.

The increase in throughput can be analyzed in DPPH protocol is due to increase in PDR and decrease in the loss and delay. The enhancement in the PDR, and Throughput and the reduction in PLR and Delay show the effectiveness of the proposed protocol and we analyze that, DPPH is capable of strengthening the performance of the healthcare WBAN system.

\section{CONCLUSION}

Proposed Healthcare WBAN systems will provide timely delivery of health data at anytime and anywhere. This study reviewed the state-of-the-art on healthcare projects and protocols. Based on this compressive review, we believe that Healthcare services and applications already has a very important and determinant role in the renovation of old healthcare services and systems that still based on the physical relationship between patient and physician or medical person. 
Moreover, proposed system has a strong impact on all expected parameters, such as packet delivery ratio, loss ratio delay, throughput, and emergencies treatment.

\section{REFERENCES}

[1] D. Malan et al., "CodeBlue: An Ad-Hoc Sensor Network Infrastructure for Emergency Medical Care", In Proceedings of the MobiSys, Workshop on Applications of Mobile Embedded Systems (WAMES 2004), Boston, MA, USA, pp.6-9, June 2004.

[2] A. Halteren et al., "Mobile Patient Monitoring: The MobiHealth System", J. Inform. Tech. Healthcare, vol. 2, pp. 365-373, 2004.

[3] J. W. P. Ng et al., "Ubiquitous Monitoring Environment for Wearable and Implantable Sensors (UbiMon)". In Proceedings of 6th International Conference on Ubiquitous Computing (UbiComp'04), Nottingham, UK, pp. 7-14, September 2004.

[4] A. Wood et al., "ALARM-NET: Wireless Sensor Networks for Assisted-Living and Residential Monitoring”, Technical Report CS-2006-01, Department of Computer Science, University of Virginia, Charlottesville, VA, USA, 2006.

[5] J. Ko et al., "MEDiSN: Medical Emergency Detection in Sensor Networks. ACM Transaction on Embedded Computer System, vol. 10, pp.1-29, 2010.

[6] Madhumita Kathuria and Dr. Sapna Gambhir, "Quality of service provisioning transport layer protocol for WBAN system", International Conference on Optimization, Reliability and Information Technology (ICROIT, IEEE Xplore), pp. 222-228, 2014.

[7] C. Wang et al., "Priority-based Congestion Control in Wireless Sensor Networks", Proceedings of the IEEE International Conference on Sensor Networks, Ubiquitous, and Trustworthy Computing (SUTC'06), 2006.

[8] C. Wang et al.,"Upstream congestion control in wireless sensor networks through cross-layer optimization", IEEE journal on selected area in communication, vol. 25 , no. 4 , pp. 786-795, 2007.

[9] S. Misra et al., "LACAS: Learning Automata-Based Congestion Avoidance Scheme for Healthcare Wireless Sensor Networks", IEEE Journal on Selected Areas in Communications, pp. 466-479, 2009.

[10] Nazbanoo Farzaneh Bahalgardi. et al., "An Adaptive Congestion Alleviating Protocol for Healthcare Applications in Wireless Body Sensor Networks: Learning Automata Approach", Amirkabir, Electrical \&
Electronics Engineering, Springer 2012, vol. 44, no. 1, pp.31-41, 2012

[11] M. H. Yaghmaee et al., "A Prioritization Based Congestion Control Protocol for Healthcare Monitoring Application in Wireless Sensor Networks", Springer, Wireless Pers Commun, PP. 2605-2631, 2013.

[12] Abbas Ali Rezaee et al., "Optimized Congestion Management Protocol for Healthcare Wireless Sensor Networks", Wireless Pers Commun, (C) Springer, pp. 1134, 2014.

[13] Sapna Gambhir, Vrisha Tickoo, and Madhumita Kathuria,"Priority based congestion control in WBAN", Eighth International conference on contemporary computing (SCOPUS, DBLP, IEEE Xplore), pp. 428433, 2015.

[14] M. Kathuria, and S. Gambhir, " Reliable Delay Sensitive Loss Recovery Protocol for critical Health data Transmission System", 1st International Conference on Futuristic trend in Computational Analysis and Knowledge Management (IEEE Xplore), PP. 333-339, 2015.

[15] Sapna Gambhir and Madhumita Kathuria, "DWBAN: Dynamic Priority based WBAN Architecture for Healthcare System", 3rd International Conference on Computing for Sustainable Global Development (IndiaCom-2016, IEEE Xplore), pp. 6133-6139, 2016.

[16] Madhumita Kathuria and Dr. Sapna Gambhir, "Leveraging machine learning for optimize predictive classification and scheduling E-Health traffic", International Conference on Recent Advances and Innovations in Engineering (IEEE Xplore), pp. 1-7, 2014.

[17] H. Cao et al., "Employing IEEE802.15.4 for quality of service provisioning in wireless body area networks", In Proceedings IEEE advanced information networking and application, AINA 2010.

[18] Madhumita Kathuria and Dr. Sapna Gambhir, "Genetic Binary Decision Tree based Packet Handling schema for WBAN system", Recent Advances in Engineering and Computational Sciences (IEEE Xplore), pp. 1-6, 2014.

[19] G. Zhou et al., "BodyQoS: Adaptive and Radio-Agnostic QoS for Body Sensor Networks", In Proceedings of IEEE INFOCOM, 2008.

[20] Vrisha Tickoo and Sapna Gambhir, " A Comparison study of Congestion Control Protocols in WBAN" in proceeding of International Journal of Innovations \& Advancement in Computer Science (IJIACS), vol. 4, no. 6, pp. 121-127, June 2015. 